\title{
miR663a-TTC22V1 axis inhibits colon cancer metastasis
}

\author{
WEI TIAN*, YANTAO DU*, YUWAN MA*, BAOZHEN ZHANG, LIANKUN GU, \\ JING ZHOU and DAJUN DENG
}

\begin{abstract}
Key Laboratory of Carcinogenesis and Translational Research (Ministry of Education/Beijing), Division of Etiology, Peking University Cancer Hospital and Institute, Beijing 100142, P.R. China
\end{abstract}

Received May 4, 2018; Accepted January 10, 2019

DOI: $10.3892 /$ or.2019.6969

\begin{abstract}
An increasing number of studies have demonstrated that microRNAs (miRs) may act as oncogenes or anti-oncogenes in various types of cancer, including colon cancer (CC). However, the clinical and biological significance of miR663a in the prognosis of $\mathrm{CC}$ and its underlying molecular mechanisms remain unknown. Using the reverse transcription-quantitative polymerase chain reaction on $\mathrm{CC}$ and surgical margin tissue samples from 172 patients with CC, it was identified that miR663a was significantly downregulated in $\mathrm{CC}(\mathrm{P}<0.001)$, particularly in metastatic $\mathrm{CC}(\mathrm{P}=0.044)$. miR663a overexpression inhibited the proliferation and migration/invasion of $\mathrm{CC}$ cells in vitro, and also tumor growth and metastasis of CC cells in vivo. Additionally, miR663a target genes were analyzed. Inverse changes in tetratricopeptide repeat domain 22 variant 1 (TTC22VI) in response to alterations in miR663a expression were observed. miR663a decreased the reporter activity of the wild-type TTC22VI-3' untranslated region (UTR), but did not decrease that of a 3'UTR mutant. miR663a completely abolished cell migration/invasion induced by TTC22VI containing the wild-type 3'UTR sequence, but not that induced by TTC22VI containing the 3'UTR mutant. An inverse correlation between miR663a and TTC22 mRNA levels was observed in $\mathrm{CC}$ tissues. These results suggest that
\end{abstract}

Correspondence to: Professor Dajun Deng, Key Laboratory of Carcinogenesis and Translational Research (Ministry of Education/Beijing), Division of Etiology, Peking University Cancer Hospital and Institute, 52 Fu-Cheng-Lu, Beijing 100142, P.R. China E-mail: dengdajun@bjmu.edu.cn

${ }^{*}$ Contributed equally

Abbreviations: CC, colon cancer; TTC22V1, tetratricopeptide repeat domain 22 variant 1; SM, surgical margin; UTR, untranslated region; 3'UTR-wt, wild-type TTC22VI 3'UTR; 3'UTR-mut, 3'UTR-mutant control; TTC22VI\&3'UTR-wt, TTC22V1 cDNA sequence containing a wild-type 3'UTR; TTC22V1\&3'UTR-mut, TTC22VI cDNA sequence containing a 3'UTR mutant

Key words: microRNA-663a, tetratricopeptide repeat domain 22 variant 1 , colon cancer, metastasis, overall survival time
TTC22V1 mRNA is a crucial miR663a target that directly promotes cell migration/invasion. TTC22, which, to the best of our knowledge, has rarely been investigated, is located in the nuclei of epithelial cells in colon stem cell niches at crypt bases, and is significantly downregulated in CC, particularly in non-metastatic CC. High TTC22VI expression is a significant poor survival factor for patients with CC. Collectively, the results of the present study suggested that TTC22VI may be a metastasis-associated gene and that the miR663a-TTC22VI axis inhibited $\mathrm{CC}$ metastasis.

\section{Introduction}

Primate-specific microRNA-663a ( $m i R 663 a)$ is associated with a number of biological processes, including viral infection and autoimmune diseases, particularly inflammation (1-6). Inflammation contributes to cancer development and progression. However, the effects of miR663a on cancer development remain poorly understood. The reported roles of miR663a in cancer development and progression are contradictory. Whereas miR663a may promote malignancy in prostate and nasopharyngeal cancers (7-9), it may also suppress brain and pancreatic cancers (10-12), suggesting organ-specific roles for miR663a in cancer development. We recently identified that metastasis-associated lung adenocarcinoma transcript 1 , a competing endogenous RNA, is a master regulator of miR663a through a direct microRNA (miRNA)-long non-coding RNA interaction (13). A number of miR663a target genes (e.g. APC, CDKN2A, CXCR4, eEF1A2, $H M G A 2$, JUND, CDKNIA and TGFBI) have been reported in various cell lineages (4,7-16); however, whether the expression patterns of these target genes contribute to the organ-specific roles of miR663a in cancer development remains unclear.

Tetratricopeptide repeat (TPR) domain 22 (TTC22) is a gene that has rarely been investigated (17). TPR motifs are present in a variety of proteins. The majority of TPR motifs are associated with multi-protein complexes. There is extensive evidence indicating that TPR motifs are important for functions associated with the cell cycle, transcription, protein transport complexes and other protein-protein interactions $(18,19)$. According to the Human Protein Atlas (20), the TTC22 gene is expressed comprehensively in epithelial cells in the normal gastrointestinal tract mucosa, but is lacking in gastrointestinal cancer tissues, suggesting a possible role for TTC22 in the development and progression of colon cancer (CC). 
Bioinformatics analysis results indicated that TTC22 variant 1 (TTC22V1) mRNA may be an miR663a candidate target. In the present study, alterations in miR663a and TTC22V1 expression during CC development and their association with $\mathrm{CC}$ progression were investigated. Most importantly, for the first time, to the best of our knowledge, TTC22V1 is identified as a key miR663a target and that the miR663a-TTC22VI axis serves a crucial role in promoting CC metastasis.

\section{Materials and methods}

Patients and tissue samples. $\mathrm{CC}$ and paired non-cancerous surgical margin (SM; $>5 \mathrm{~cm}$ from the cancer mass) samples were collected from 172 inpatients (average age, 61.6 years; 101 males and 71 females; 89 patients with $\mathrm{CC}$ at pathological tumor-node-metastasis stage I-II (21) and 83 patients with CC at stage III-IV) beTween-2004 and 2011 at the Biological Sample Bank, Peking University Cancer Hospital and Institute, Beijing, China (Table I). Clinicopathological and 3-year follow-up data were available for all patients.

$R N A$ extraction and reverse transcription-quantitative polymerase chain reaction ( $R T-q P C R)$. In total, $50 \mathrm{ng}$ RNA was extracted from fresh tissue samples or cell lines using TRIzol ${ }^{\circledR}$ reagent (Thermo Fisher Scientific, Inc., Waltham, MA, USA), according to the manufacturer's protocol $\left(25^{\circ} \mathrm{C}\right.$ for $10 \mathrm{~min}$ and $42^{\circ} \mathrm{C}$ for $\left.30 \mathrm{~min}\right)$. The quality and concentration of the RNA samples were determined using a NanoDrop 2000 system (Thermo Fisher Scientific, Inc.). The amount of mature miR663a (Entrez Gene accession no. 724033) transcripts was determined using a Bulge-Loop ${ }^{\mathrm{TM}}$ miRNA RT-qPCR starter kit (cat. no. 10211; Guangzhou RiboBio Co., Ltd., Guangzhou, China) and a Bulge-Loop hsa-miR-663a RT-qPCR primer set (miRQ0003326-1; Guangzhou RiboBio Co., Ltd.), according to the manufacturer's protocol. U6 RNA was used as a RT-qPCR reference. The thermocycling conditions were 40 cycles of $95^{\circ} \mathrm{C}$ for $2 \mathrm{sec}, 62^{\circ} \mathrm{C}$ for $20 \mathrm{sec}$ and $72^{\circ} \mathrm{C}$ for $30 \mathrm{sec}$ for miR663a and U6. TTC22V1 (Entrez gene accession no. 55001) expression was determined using a StepOne Real-Time PCR system (Applied Biosystems; Thermo Fisher Scientific, Inc.) and SYBR-Green PCR master mix reagent (FastStart Universal SYBR-Green Master; Roche Diagnostics GmbH, Mannheim, Germany), according to the manufacturer's protocol with forward primer, 5'-atccacatcagagcctacctg-3' and reverse primer, 5'-cgtccacgcccatatagtagt-3'. Gene expression levels were normalized to those of $G A P D H$ (forward primer, 5'-gaaggtgaaggtcggagt-3' and reverse primer, 5'-gaggatggtgatgggattc- $3^{\prime}$ ) or to those of $A L U$ (forward primer, 5'-gaggctgaggcaggagaatcg-3' and reverse primer, 5'-gtcgcccaggctggagtg- $3^{\prime}$ ) as in the previous correlation analysis (22). The thermocycling conditions were 40 cycles of $95^{\circ} \mathrm{C}$ for $15 \mathrm{sec}$, $58^{\circ} \mathrm{C}$ for $20 \mathrm{sec}$ and $72^{\circ} \mathrm{C}$ for $30 \mathrm{sec}$ for TTC22V1, GAPDH and $A L U$. Relative mRNA levels were calculated using the $\Delta \Delta \mathrm{Cq}$ method (23). Each sample was evaluated in triplicate.

Cell lines, culture and authentication. The HCT116 and SW480 cancer cell lines were purchased from the American Type Culture Collection (Manassas, VA, USA). The RKO cell line was kindly provided by Dr Guoren Deng
(University of California San Francisco, San Francisco, CA, USA). The cell lines were cultured in RPMI-1640 medium containing $10 \%$ fetal bovine serum (FBS) and $100 \mathrm{U} / \mathrm{ml}$ penicillin/streptomycin (Thermo Fisher Scientific, Inc.) at $37^{\circ} \mathrm{C}$ in a humidified incubator containing $5 \% \mathrm{CO}_{2}$. The $\mathrm{Caco} 2$ cell line was purchased from the National Infrastructure of Cell Line Resource (Beijing, China), and was cultured in minimal essential medium containing $10 \%$ FBS and $1 \%$ non-essential amino acids (Thermo Fisher Scientific, Inc.). All cell lines were tested and authenticated using the Goldeneye20A STR Identifiler PCR Amplification kit (Beijing Jianlian Genes Technology Co., Ltd., Beijing, China) prior to use in the study.

Plasmids, oligonucleotides and transfection. miR663a mimic (forward, 5'-aggcggggcgccgcgggaccgc-3' and reverse, 5'-gguccegcggegceccgecuuu-3'); and antisense/inhibitor (5'-gcggucccgeggcgeccegccu-3') products were synthesized by Shanghai GenePharma Co., Ltd. (Shanghai, China). A scrambled short interfering RNA set (dsR-Ctrl; forward, 5'-uucuccgaacgugucacgutt-3' and reverse, 5'-acgugacacguucggagaatt-3') was used as the mimic negative control. A scrambled RNA (ssR-Ctrl; 5'-caguacuuuuguguaguacaa-3') was used as the inhibitor negative control (13).

An miR663a expression vector, pcDNA3.1b_pri-miR663a, was constructed with $93-\mathrm{bp}$ PCR products $\left(95^{\circ} \mathrm{C}\right.$ for $30 \mathrm{sec}$, $54^{\circ} \mathrm{C}$ for $30 \mathrm{sec}$, and $72^{\circ} \mathrm{C}$ for $50 \mathrm{sec}$ for 35 cycles) amplified from A549 cell genomic DNA using HiFi DNA polymerase, forward primer, 5'-ccttccggcgtcccaggcg-3' and reverse primer, 5'-catggccgggccaccaggaaa-3'. Green fluorescent protein (GFP)-tagged miR663a (GFP-miR663a, 5'-aggcggggcgccgcgggaccgc-3') expression vectors were purchased from Shanghai GenePharma Co., Ltd. for stable transfection. A scrambled RNA (5'-aaatgtactgcgcgtggagac-3') expression vector was used as a negative control (13).

The wild-type TTC22V1 3' untranslated region (UTR) (3'UTR-wt) plasmid was cloned from A549 cells using forward primer, 5'-gatctctagagtgcggetgetcttcgagaccat-3' and reverse primer, 5-atgctctagagtcacaattcccgaccaagaatcgaa-3'. The 3'UTR-mutant control (3'UTR-mut) plasmid was constructed using a site mutation kit (Agilent Technologies, Inc., Santa Clara, CA, USA). A TTC22V1 expression vector, pCMV6-Entry_TTC22V1, was purchased from OriGene Technologies, Inc. (cat. no. RC225966; Rockville, MD, USA). Wild-type and mutant 3'UTR sequences were synthesized and inserted into the 3'-terminus of the TTC22VI DNA sequence in the expression vectors (TTC22V1\&3'UTR-wt and $T T C 22$ V1\&3'UTR-mut, respectively) to investigate the contribution of $T T C 22 \mathrm{VI}$ to the inhibition of CC development by miR663a.

The plasmids or oligonucleotides (final concentration, $1 \mu \mathrm{g} / \mathrm{ml}$ ) were transfected using X-tremeGENE ${ }^{\mathrm{TM}}$ HP DNA transfection reagent (Roche Diagnostics GmbH). At $24 \mathrm{~h}$ post-transfection, the medium was replaced with RPMI-1640 containing $10 \%$ FBS. The entire process was performed according to the manufacturer's protocol.

Western blotting. Western blotting was performed as previously described (24). Briefly, HCT116, RKO and MKN45 cells $\left(1.5 \times 10^{6}\right)$ were harvested at $500 \mathrm{x} \mathrm{g}$ for $5 \mathrm{~min}$. Proteins 
were extracted and diluted in $200 \mu \mathrm{l} 1 \mathrm{X}$ cell lysis buffer [50 mmol/1 Tris/HCl (pH 6.8), $100 \mathrm{mmol} / 1$ DTT, 2\% SDS, $0.1 \%$ bromophenol blue and $10 \%$ glycerol] and $20 \mu \mathrm{l}$ protein lysate was loaded per lane. Proteins were separated by SDS-PAGE (10\% gel). The following primary antibodies were used: Goat anti-TTC22 polyclonal antibody $\left(4^{\circ} \mathrm{C} ; 1: 1,000\right.$; cat. no. sc-249130; Santa Cruz Biotechnology, Inc., Dallas, TX, USA), and mouse anti-GAPDH monoclonal antibody $\left(4^{\circ} \mathrm{C} ; 1: 10,000\right.$; cat. no. 97166; Cell Signaling Technology, Inc., Danvers, MA, USA). The horseradish peroxidase-conjugated anti-mouse or -goat secondary antibody (cat. no. ZB-2306 or ZB-2305; ZhongShan Jinqiao Biotechnology, Co., Ltd. Beijing, China) was incubated at room temperature for $45 \mathrm{~min}$ at a dilution of 1:2,000 in 1\% fat-free milk. For tissue samples, proteins from $50 \mathrm{mg}$ tissue were extracted and diluted in $500 \mu \mathrm{l} 1 \mathrm{X}$ cell lysis buffer. Other processes were the same as for cell samples.

Immunohistochemistry staining (IHC). A rabbit anti-TTC22 polyclonal antibody (1:500; cat. no. HPA035072, specific for TTC22V1; Sigma-Aldrich; Merck KGaA, Darmstadt, Germany) was used for the IHC analysis. The Dako REAL ${ }^{\mathrm{TM}}$ EnVision $^{\mathrm{TM}}$ Detection system (peroxidase/diaminobenzidine+, rabbit/mouse; Dako; Agilent Technologies, Inc.) was used to visualize the primary antibody-binding cells according to the manufacturer's protocol. Briefly, paraffin sections $(4 \mu \mathrm{m})$ were dewaxed and rehydrated in xylene and ethanol. The sections were autoclaved in a $10 \mathrm{mM}$ sodium citrate buffer containing $0.05 \%$ Tween-20 (pH 6.0) for 3 min for antigen retrieval and then immersed in $3 \% \mathrm{H}_{2} \mathrm{O}_{2}$ for 10 min to block endogenous peroxidase. Following submerging in 5\% bovine serum albumin (BSA) (cat. no. A1933; Sigma Life Science; Merck KGaA) for $60 \mathrm{~min}$, the sections were incubated with the primary antibody overnight at $4^{\circ} \mathrm{C}$. The PBS-washed sections were then treated with the Dako REAL ${ }^{\mathrm{TM}}$ EnVision $^{\mathrm{TM}}$ Detection system and counterstained with hematoxylin (0.125\%; ZhongShan Jinqiao Biotechnology, Co., Ltd.) at room temperature for $1 \mathrm{~min}$. Normal rabbit IgG (cat. no. ZDR-5118; ZhongShan Jinqiao Biotechnology, Co., Ltd.) was used as the negative control (diluted and incubated as for the anti-TTC22 antibody).

Confocal microscopic determination of TTC22V1 protein expression. TTC22V1 protein expression in Caco2, HCT116 and SW480 cells was analyzed using the aforementioned rabbit anti-TTC22 polyclonal antibody. The rabbit IgG antibody was used as a negative control. Briefly, HCT116, SW480 and Caco2 cells were cultured on coverslips and fixed with $10 \%$ paraformaldehyde for $10 \mathrm{~min}$ at room temperature, followed by permeabilization with $0.5 \%$ Triton X-100 in PBS for $15 \mathrm{~min}$ and blocking with $5 \%$ BSA overnight at $4{ }^{\circ} \mathrm{C}$. Following incubation with the anti-TTC22V1 antibody (1:500) or the rabbit $\mathrm{IgG}$ negative control antibody for $2 \mathrm{~h}$ at room temperature, the cells were probed with a fluorescein isothiocyanate-conjugated secondary antibody (1:100 in PBS at room temperature for 45 min; cat. no. ZF-0311; ZhongShan Jinqiao Biotechnology, Co., Ltd.), counterstained with DAPI $(1 \mu \mathrm{g} / \mathrm{ml})$ at room temperature for $10 \mathrm{~min}$, and mounted with $50 \%$ glycerol in PBS. A Leica SP2 confocal system (Leica Microsystems $\mathrm{GmbH}$, Wetzlar, Germany) was used to observe the localization of TTC22V1.
Cell proliferation and migration assays using the IncuCyte platform. CC cell HCT116 and SW480 cells, whose biological behavior was significantly affected by miR663a expression changes (13), were seeded into 96-well plates (3,000 cells/well; 6-wells/group) and cultured at $37^{\circ} \mathrm{C}$ for at least $96 \mathrm{~h}$ to generate proliferation curves. Images were captured of the cells every $2 \mathrm{~h}$. Cell confluence was analyzed with IncuCyte ZOOM software 2015A (Essen Bioscience, Ann Arbor, MI, USA). For the continuous observation of cell migration and invasion, cells were seeded into 96-well plates at a density of 10,000 cells/well and cultured for $24 \mathrm{~h}$. Subsequently, a wound was made in the cell layer, and the cells were washed 3 times with PBS. The cells were regularly cultured and photographed every $2 \mathrm{~h}$ for at least $96 \mathrm{~h}$. The relative wound density and the width were calculated using the same software.

Transwell invasion assay. Plates (24-well) with Transwell permeable supports with a $6.5 \mathrm{~mm}$ insert and an $8.0 \mu \mathrm{m}$ polycarbonate membrane (Corning, Inc., Corning, NY, USA) were used in the cell invasion assay. The upper chamber was pre-coated with a $100 \mu \mathrm{l}$ mixture of BD Matrigel (BD Biosciences, San Jose, CA, USA) and RPMI-1640 (1:5, v/v). The lower chamber contained $800 \mu 1$ RPMI-1640 medium containing 10\% FBS. Cells were seeded into the upper chamber $(3,000$ cells/well, 3 -wells/group). After 24 or $36 \mathrm{~h}$ of incubation at $37^{\circ} \mathrm{C}$, the $6.5-\mathrm{mm}$ inserts were removed from the plate, fixed in $4 \%$ paraformaldehyde, and stained with a crystal violet solution ( $0.1 \%$; at room temperature for $30 \mathrm{~min})$. Non-invasive cells on the upper surface of the insert were wiped away with a cotton swab. The number of invaded cells was counted manually in 4 randomly selected fields under a light microscope. All experiments were performed at least 3 times.

Dual-luciferase reporter assay. The wild-type TTC22VI 3'UTR was inserted into the pGL-control vector (cat. no.E1741; Promega Corporation, Madison, WI, USA) and used to transfect HCT116 cells in a 24-well plate using X-tremeGENE ${ }^{\text {TM }}$ HP DNA transfection reagent (Roche Diagnostics GmbH). Pre-miR663a or the control vector was co-transfected with the Renilla vector (3-wells/group). The activities of both Renilla and firefly TTC22V1 3'UTR-wt/-mut luciferases were determined using a Dual-Luciferase Reporter Assay system (Promega Corporation) at $72 \mathrm{~h}$ post-transfection.

RNA pulldown assay. HCT116 cells $\left(3 \times 10^{6}\right)$ seeded in a $10-\mathrm{cm}$ plate for $24 \mathrm{~h}$ were transfected with biotin-labeled miR-NC (forward, 5'-uucuccgaacgugucacgutt-3' and reverse, 5'-acgugacacguucggagaatt-3'); biotin-labeled miR663a-wt (forward, 5'-aggcggggcgccgcgggaccgc-3' and reverse, 5'-gcggucccgcggcgeccegccu-3'); or biotin-labeled miR663a-mut (forward, 5'-cuuacauucgccgegggaccgc-3' and reverse, 5'-gcggucccgcggcgaauguaag-3') (final concentration, $100 \mathrm{nM}$ ). The cell lysates were harvested at $48 \mathrm{~h}$. Streptavidin Dynabeads (Dynabeads ${ }^{\circledR}$ M-280 Streptavidin; cat. no. 11205D; Thermo Fisher Scientific, Inc.) $(50 \mu 1 /$ pulldown) were coated with $10 \mu 1$ yeast tRNA (stock, $10 \mathrm{mg} / \mathrm{ml}$; Ambion; Thermo Fisher Scientific, Inc.), $10 \mu \mathrm{l} \mathrm{BSA}(10 \mathrm{mg} / \mathrm{ml}$ stock) and $480 \mu 1$ lysis buffer and incubated with rotation at $4^{\circ} \mathrm{C}$ for $30 \mathrm{~min}$. The beads were washed and mixed with sample lysates (600 $\mu 1 /$ pulldown) and incubated overnight at $4^{\circ} \mathrm{C}$ on a rotator. The beads were then 
pelleted the next day to remove the unbound materials and were digested with $5 \mu \mathrm{l}$ RNase-free DNase I $(2 \mathrm{U} / \mu \mathrm{l})$ at $37^{\circ} \mathrm{C}$ for $10 \mathrm{~min}$ and $5 \mu \mathrm{l}$ proteinase $\mathrm{K}(10 \mathrm{mg} / \mathrm{ml})$ at $55^{\circ} \mathrm{C}$ for $20 \mathrm{~min}$. Subsequently, together with $50 \mu 1$ input, RNA was extracted, and RT-qPCR was used to detect the target TTC22V1 mRNA as aforementioned.

Tumor implantation and experimental metastasis. Non-obese diabetic (NOD)-severe combined immunodeficient (SCID) mice ( $\mathrm{n}=30$; female, 6-8 weeks, 18-20 g) were purchased from Beijing HFK Bioscience Co., Ltd. (Beijing, China) and were allowed to acclimatize for 1 week before use. Housing conditions were $25^{\circ} \mathrm{C}$, humidity $45-55 \%, 1$ atm pressure, $12-\mathrm{h}$ light/12-h dark cycle, free choice feeding, water ad libitum. Cells stably transfected with the GFP-miR663a and control vectors were digested with trypsin. A cell suspension $\left(1 \times 10^{7}\right.$ cells $\left./ \mathrm{ml}\right)$ was injected subcutaneously $(150 \mu \mathrm{l} / \mathrm{site}$, $10 \mathrm{mice} /$ group) into the left and right legs of NOD-SCID mice, respectively. At 3 weeks after implantation, the mice were sacrificed and the weights of their xenograft tumors were determined.

To examine experimental metastasis, $1.25 \times 10^{6}$ HCT116 cells stably transfected with the GFP-miR663a or control vector were injected into the tail veins of NOD-SCID mice (10 mice/group). After 2 months, the mice were sacrificed and dissected. Metastatic nodules in the lung and liver surfaces were counted. Tissues were fixed in formalin and prepared to create $4-\mu \mathrm{m}$ paraffin-embedded slices. The slices were stained with $0.125 \%$ hematoxylin and $1 \%$ eosin at room temperature for $10 \mathrm{~min}$ and observed via light microscopy.

miR663a target selection. The list of possible miR663a target candidates was downloaded from the miRNAMap website (mirnamap.mbc.nctu.edu.tw/php/mirna_entry. php?acc $=$ MI0003672) and obtained from the National Center for Biotechnology Information (NCBI) website (blast.ncbi. nlm.nih.gov) through searching the miR663a matched mRNA-3'UTRs in the human genome and transcripts. When downregulation (or upregulation) of target gene expression was $>1.5$-fold in HCT116 cells $72 \mathrm{~h}$ after transient transfection of miR663a expression vector (or antisense/inhibitor treatment) in the Affymetrix U133 Plus 2.0 cDNA array datasets (NCBI Gene Expression Omnibus accession no. GES117918) (13), it was selected as the candidate target.

Statistical analyses. All statistical analyses were performed using SPSS software (version 18.0; SPSS, Inc., Chicago, IL, USA). The Shapiro-Wilk test was used to estimate the normality of the distributions. Student's t-test or one-way analysis of variance was used for normally distributed data. Patient overall survival times were analyzed using the Kaplan-Meier method and log-rank test. Cox's proportional hazard model was used for multivariate analyses. Differences in miR663a and TTC22V1 expression levels were assessed using the Mann-Whitney $U$ test or Wilcoxon signed-rank test. Spearman's non-parametric analyses were performed to determine the correlation between gene expression. $\mathrm{P}<0.05$ was considered to indicate a statistically significant difference. All experiments were performed at least 3 times with samples in triplicate.

\section{Results}

Downregulation of miR663a expression in CC tissues. To the best of our knowledge, the expression status of miR663a in $\mathrm{CC}$ tissues has not been reported previously. Using qPCR analysis, it was identified that the relative miR663a expression levels in CC tissues were significantly decreased compared with those in SM tissues from 172 patients (median, 0.263 vs. 0.555; Mann-Whitney U test, $\mathrm{P}=0.001$; Fig. $1 \mathrm{~A}$ ). In addition, the miR663a expression levels in metastatic CC tissues were also significantly decreased compared with those in non-metastatic CC tissues ( $\mathrm{P}=0.044$; Fig. $1 \mathrm{~B}$ and Table I). No significant association between miR663a expression and age, sex, vascular embolus, CC differentiation and location was identified. The downregulation of miR663a expression in CC tissues, particularly in metastatic $\mathrm{CC}$ tissues, indicated that miR663a may serve an important role in carcinogenesis and metastasis in CC.

Inhibition of cancer cell proliferation and metastasis by miR663a in vitro and in vivo. To investigate the effects of miR663a overexpression on CC cell proliferation, migration and invasion, cells were transiently transfected with the pre-miR663a expression vector and its control vector pcDNA3.1b. Forced miR663a overexpression significantly inhibited proliferation, migration and invasion in HCT116 cells (Fig. 2A and B) and SW480 cells (Fig. 2C and D).

To validate the in vitro results, the GFP-miR663a vector was used to stably transfect HCT116 cells (Fig. 3A). The growth of HCT116 xenografts (tumors) in NOD-SCID mice was significantly decreased by miR663a overexpression $(\mathrm{P}<0.001$; Fig. 3B). GFP-miR663a tumors were observed in only $7 / 10$ mice, whereas GFP-Ctrl tumors occurred in 10/10 mice. The miR663a expression levels in GFP-miR663a tumors were $\sim 2$-fold higher compared with those in the GFP-Ctrl tumors (Fig. 3C, left).

An experimental pulmonary metastasis model of NOD-SCID mice was used to determine whether miR663a overexpression could inhibit cancer metastasis. At 2 months after injection of HCT116 cells stably overexpressing miR663a into the tail veins of mice (miR663a group), the average number of metastatic nodules on the lung surfaces in the miR663a group was significantly lower compared with in the Ctrl group (median, 1.2 vs. 6.1; Mann-Whitney U test; $\mathrm{P}=0.0383$; Fig. 3D, left). The average lung weight, which correlates positively with the number of metastatic nodules, was also lower in the miR663a group compared with in the Ctrl group (median, 0.430 vs. 0.575 g; Mann-Whitney U test; $\mathrm{P}=0.041$; Fig. 3D, right). In addition, metastatic nodules were observed on the lung surfaces of $50 \%(5 / 10)$ of the mice, whereas the lung metastatic rate was $70 \%(7 / 10)$ in mice injected with the cells stably transfected with the control vector (Ctrl group), including 2 giant metastatic lesions on the lung hilus (Fig. 3E, white arrow). These results indicate that miR663a overexpression inhibits experimental metastasis in HCT116 cells in vivo.

Characterization of TTC22V1 mRNA as a crucial miR663a target. To search for candidate miR663a targets in CC tissues, a bioinformatics analysis was performed using sequence search 
Table I. Comparison of miR663a and TTC22V1 gene expression level in colon carcinoma with various clinicopathological characteristics.

\begin{tabular}{|c|c|c|c|c|c|}
\hline Characteristics & $\mathrm{n}$ & $\begin{array}{c}\text { Median relative expression } \\
\text { level of } m i R 663 a \\
\text { (interquartile range) }\end{array}$ & $\mathrm{P}$-value & $\begin{array}{l}\text { Median relative expression } \\
\text { level of } T T C 22 \mathrm{~V} 1 \mathrm{mRNA} \\
\quad \text { (interquartile range) }\end{array}$ & P-value \\
\hline Age, years & & & 0.286 & & 0.134 \\
\hline$\leq 60$ & 75 & $0.31(0.15-0.56)$ & & $0.07(0.03-0.17)$ & \\
\hline$>60$ & 97 & $0.25(0.12-0.47)$ & & $0.01(0.05-0.22)$ & \\
\hline Sex & & & 0.295 & & 0.820 \\
\hline Male & 101 & $0.29(0.15-0.49)$ & & $0.08(0.04-0.17)$ & \\
\hline Female & 71 & $0.23(0.09-0.53)$ & & $0.09(0.03-0.17)$ & \\
\hline Location & & & 0.962 & & 0.432 \\
\hline Sigmoid & 87 & $0.30(0.10-0.51)$ & & $0.08(0.03-0.18)$ & \\
\hline Others & 85 & $0.25(0.14-0.65)$ & & $0.08(0.05-0.21)$ & \\
\hline Differentiation & & & 0.065 & & 0.162 \\
\hline Poor & 39 & $0.34(0.13-0.65)$ & & $0.11(0.03-0.25)$ & \\
\hline Moderate/well & 133 & $0.25(0.11-0.49)$ & & $0.07(0.04-0.15)$ & \\
\hline Vascular embolus & & & 0.931 & & 0.076 \\
\hline Negative & 140 & $0.26(0.11-0.55)$ & & $0.07(0.03-0.16)$ & \\
\hline Positive & 32 & $0.28(0.15-0.47)$ & & $0.09(0.06-0.28)$ & \\
\hline pTNM stage & & & 0.047 & & 0.020 \\
\hline I+II & 89 & $0.33(0.12-0.71)$ & & $0.06(0.03-0.14)$ & \\
\hline III+IV & 83 & $0.25(0.13-0.35)$ & & $0.09(0.04-0.27)$ & \\
\hline Depth of invasion & & & 0.443 & & 0.286 \\
\hline $\mathrm{T}_{1-3}$ & 101 & $0.25(0.12-0.51)$ & & $0.06(0.03-0.15)$ & \\
\hline $\mathrm{T}_{4}$ & 71 & $0.32(0.14-0.52)$ & & $0.08(0.04-0.21)$ & \\
\hline Lymph metastasis & & & 0.044 & & 0.022 \\
\hline Negative $_{0}$ & 88 & $0.33(0.12-0.71)$ & & $0.06(0.03-0.14)$ & \\
\hline Positive & 84 & $0.25(0.14-0.35)$ & & $0.08(0.04-0.26)$ & \\
\hline Distant metastasis & & & 0.981 & & 0.002 \\
\hline $\mathrm{M}_{0}$ & 147 & $0.27(0.12-0.52)$ & & $0.07(0.03-0.15)$ & \\
\hline $\mathrm{M}_{1}$ & 25 & $0.25(0.14-0.51)$ & & $0.13(0.07-0.65)$ & \\
\hline
\end{tabular}

Statistical significance was determined using a Mann-Whitney U test. TTC22V1, tetratricopeptide repeat domain 22 variant 1; pTNM, pathological tumor-node-metastasis.
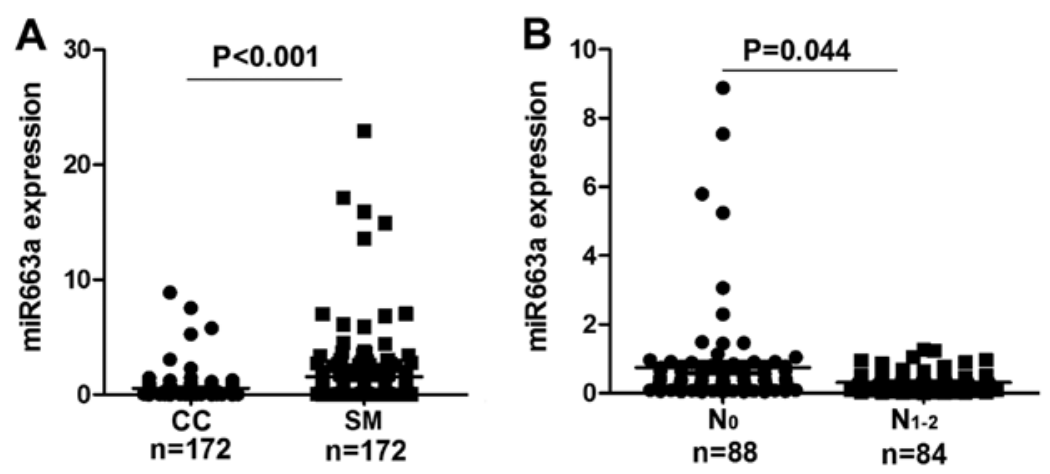

Figure 1. Comparison of miR663a expression levels in colon cancer tissue samples using the reverse transcription-quantitative polymerase chain reaction. (A) CC and the corresponding SM samples from 172 patients with CC. (B) CC with or without lymph node metastasis at the time of surgical resection. miR663a, microRNA-663a; CC, colon cancer; SM, surgical margin.

strategies. cDNA array datasets (NCBI Gene Expression Omnibus accession no. GES117918) were also used to select
miR663a target genes in HCT116 cells transiently transfected with the miR663a expression vector or its inhibitor/antisense as 
A
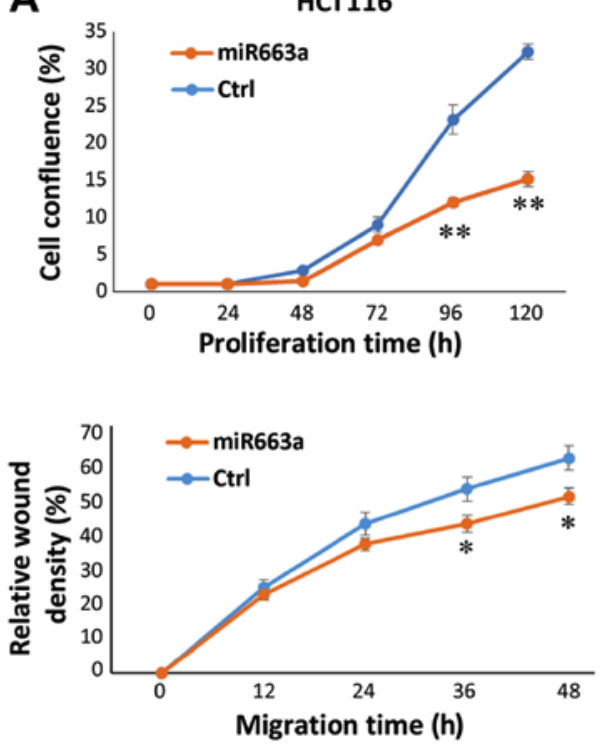

C

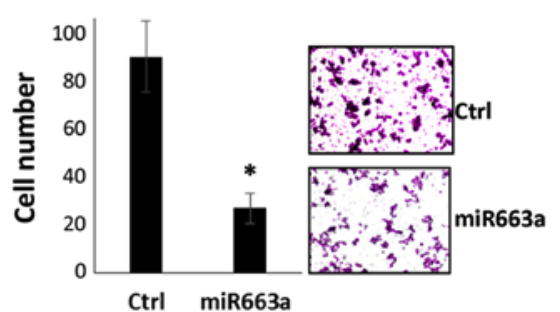

B
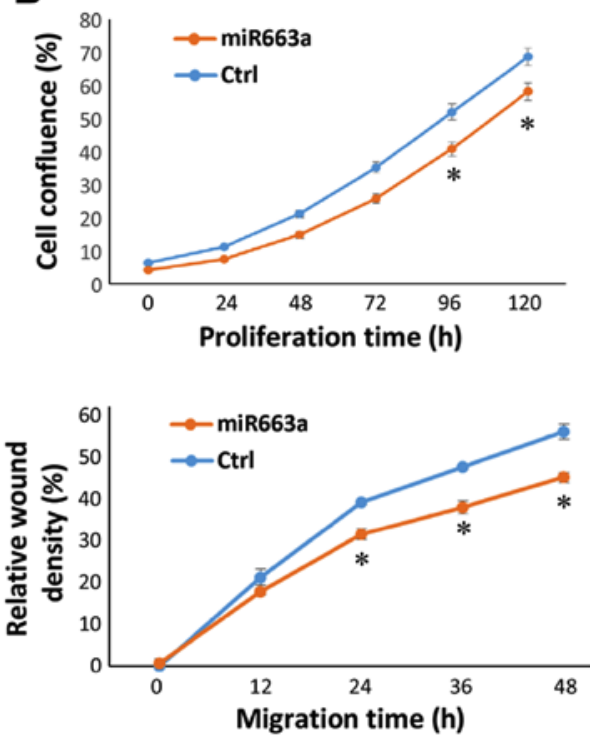

D

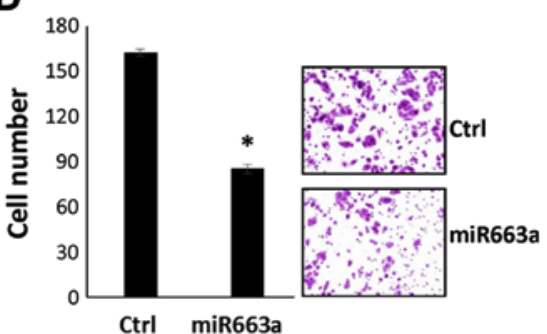

Figure 2. Effects of miR663a overexpression on the proliferation, migration and invasion of colon cancer cells. (A) Proliferation and migration curves for HCT116 cells transfected with the pre-miR663a vector according to long-term IncuCyte analyses; each point represents the mean \pm standard deviation value for 5-wells. (B) Proliferation and migration curves for SW480 cells transfected with the pre-miR663a vector according to long-term IncuCyte analyses; each point represents the mean \pm standard deviation for 5-wells. (C) Invasion of HCT116 cells transiently transfected with the pre-miR663a vector and control vector according to a typical Transwell analysis (triplicate results). (D) Invasion of SW480 cells transiently transfected with the pre-miR663a and control vector according to a typical Transwell analysis (triplicate results). ${ }^{*} \mathrm{P}<0.05 ;{ }^{* *} \mathrm{P}<0.01$ vs. Ctrl. Ctrl, pcDNA3.1b scrambled RNA control vector; $m i R 663 a$, microRNA-663a.

we reported previously (13). The bioinformatics analysis results indicated that FAM120A, KLF5, MBTD1, PSMG1, RBL1, TTC22V1, ZMYM5 and ZNF131 mRNAs may be miR663a candidate targets. However, RT-qPCR analysis results revealed that TTC22V1 mRNA was the only one whose expression level was consistently and inversely altered $>1.5$-fold following miR663a overexpression and knockdown by transient transfection with miR663a mimic and inhibitor/antisense in HCT116 cells, respectively (Fig. 4A). TTC22VI expression was downregulated in GFP-miR663a tumors (Fig. 3C, right and Fig. S1). Such an association could not be detected for FAM120A, KLF5, MBTD1, RBL1, ZMYM5 or ZNF131 (Fig. S2). Western blot analyses confirmed these results in HCT116, RKO and MKN45 cells (Fig. 4B). In the dual-luciferase assays, miR663a mimic significantly decreased the activity of the wild-type TTC22V1 3'UTR reporter (3'UTR-wt; $\mathrm{P}=0.010$ ), but not that of the 3'UTR-mut, which did not match the 5'-fragment of mature miR663a (Fig. 4C). In addition, in an miRNA pull-down assay, $T T C 22 \mathrm{~V} 1 \mathrm{mRNA}$ interacted with only wild-type miR663a and not with the miR663a mutant (Fig. 4D).

To determine the roles of TTC22VI in the inhibition of the migration and invasion of HCT116 cells by miR663a, TTC22V1\&3'UTR-wt and TTC22V1\&3'UTR-mut control vectors, integrated with full-length $T T C 22 \mathrm{VI} \mathrm{cDNA}$ and
3'UTR-wt or 3'UTR-mut control sequences, were specifically constructed and used to transiently transfect HCT116 and SW480 cells. The results of long-term dynamic IncuCyte analyses indicated that TTC22VI\&3'UTR-wt and TTC22V1\&3'UTR-mut expression significantly enhanced the migration of the cells (Fig. 5A and B). Notably, miR663a mimic transfection significantly decreased the migration of these cells transfected with TTC22V1\&3'UTR-wt, but only slightly (not significantly) decreased the migration of the cells transfected with TTC22V1\&3'UTR-mut: 15.90 vs. $4.54 \%$ for HCT116 cells at $36 \mathrm{~h}$, and 19.17 vs. $9.18 \%$ for SW480 at $60 \mathrm{~h}$ (Fig. 5A and B). The typical Transwell assay results revealed similar differences for cell invasion (Fig. 5C and D).

To determine whether the miR663a-TTC22VI axis is actually involved in CC prognosis, TTC22V1 expression levels in $172 \mathrm{CC}$ tissues and the corresponding SM tissues were determined using RT-qPCR. The results indicated that TTC22V1 mRNA levels were significantly lower in CC tissues compared with in SM tissues $(\mathrm{P}<0.001$; Fig. 6A). Western blot analyses were consistent with the RT-qPCR results (Fig. 6B). Notably, a significant reverse correlation between miR663a and TTC22V1 expression levels was also identified for $172 \mathrm{CC}$ tissues $(\mathrm{R}=-0.21$; $\mathrm{P}=0.006$; Fig. $6 \mathrm{C})$. This result further indicates that TTC22V1 is an miR663a target. 

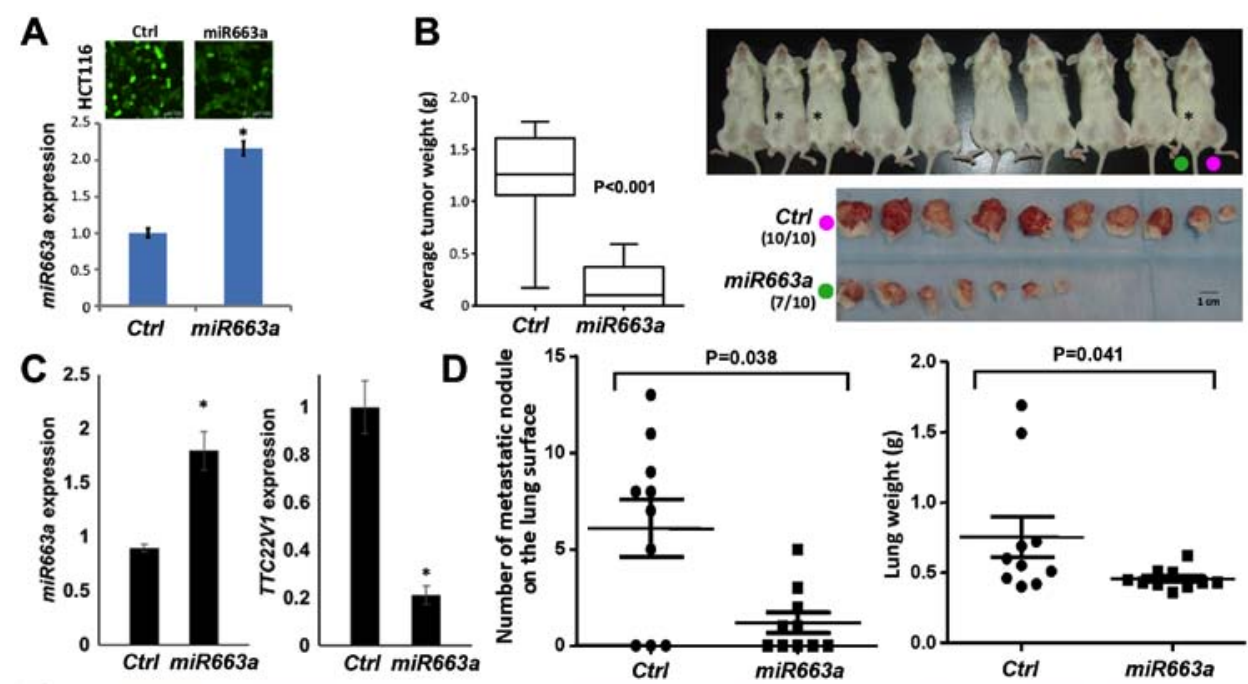

E
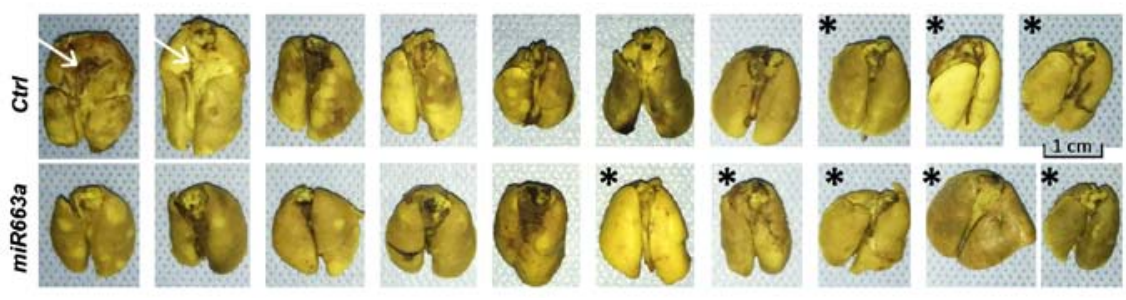

Figure 3. Effects of miR663a overexpression on the proliferation and experimental pulmonary metastasis of HCT116 cells in NOD-SCID mice. (A) miR663a expression in HCT116 cells stably transfected with the GFP-miR663a and control vectors. (B) HCT116 cell proliferation in NOD-SCID mice. Cells stably transfected with GFP-miR663a and control vector were injected subcutaneously into the left and right legs of each mouse, respectively. (C) $m i R 663 a$ and tetratricopeptide repeat domain 22 variant 1 expression levels in GFP-miR663a and GFP-Ctrl tumors determined using reverse transcription-quantitative polymerase chain reaction analysis. ${ }^{*} \mathrm{P}<0.05$ vs. Ctrl. (D) Number of metastatic nodules on the lung surface (left) and the weight of the lungs in the 2 groups of mice (right). (E) Images of the lungs with visible metastasis nodules in the miR663a stable overexpression and control groups of mice (Ctrl). The lungs without metastatic nodules are indicated with a star. Two lungs with giant metastatic lesions at the hilus in the Ctrl group are indicated with a white arrow. miR663a, microRNA-663a; NOD-SCID, non-obese diabetic-severe combined immunodeficient; GFP, green fluorescent protein; Ctrl, GFP-scrambled RNA control vector.
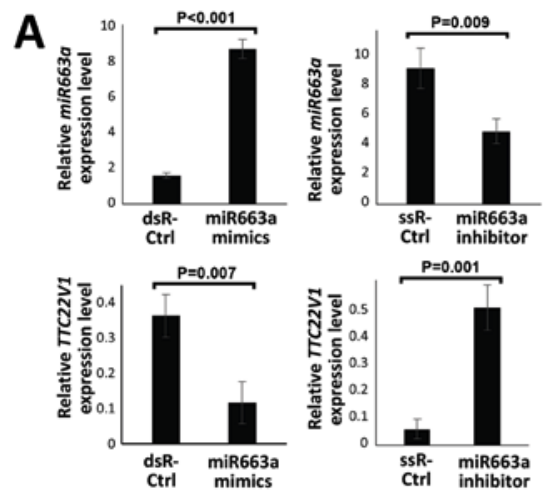

C

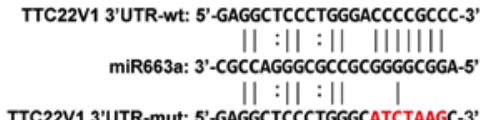

R-mut: 5'-GAGGCTCCCTGGGCATCTAAGC-3'

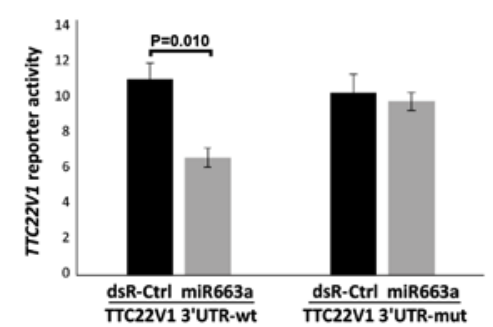

B
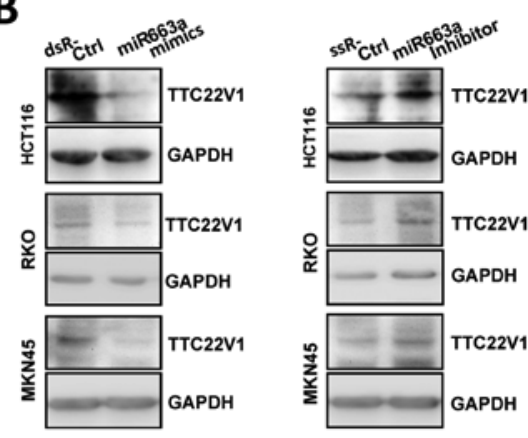

D miR663a-mut: 3'-CGCCAGGGCGCCGCTTACATTC-5' || :||:||

TTC22V1 3'UTR-wt: 5'-GAGGCTCCCTGGGACCCCGCCC-3' || :|| :|| |||||| miR663a: 3'-CGCCAGGGCGCCGCGGGGCGGA-5'

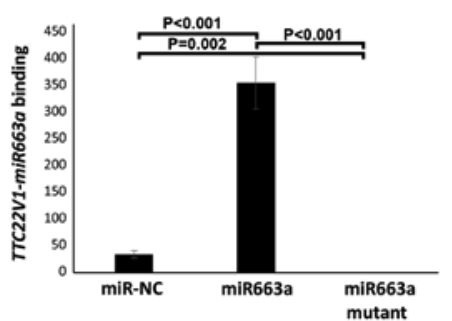

Figure 4. Association between miR663a and TTC22V1 expression levels. (A) Changes in expression levels of TTC22V1 mRNA in HCT116 cells with forced miR663a expression changes determined using reverse transcription-quantitative polymerase chain reaction analyses. (B) Western blot analysis of the amount of TTC22V1 in HCT116, RKO and MKN45 cells transiently transfected with miR663a mimic, antisense/inhibitor and negative control. (C) Dual-luciferase assay results of the effect of miR663a on the reporter activity of wild-type TTC22V1 3'UTR and its mutant in HCT116 cells. (D) Biotin-labeled miR663a pulldown assay results indicating interactions between TTC22V1 mRNA and miR663a or its mutant. miR663a, microRNA-663a; TTC22V1, tetratricopeptide repeat domain 22 variant 1; UTR, untranslated region; wt, wild-type; mut, mutant; dsR, scrambled short interfering RNA; ssR, scrambled RNA; Ctrl, control. 
A

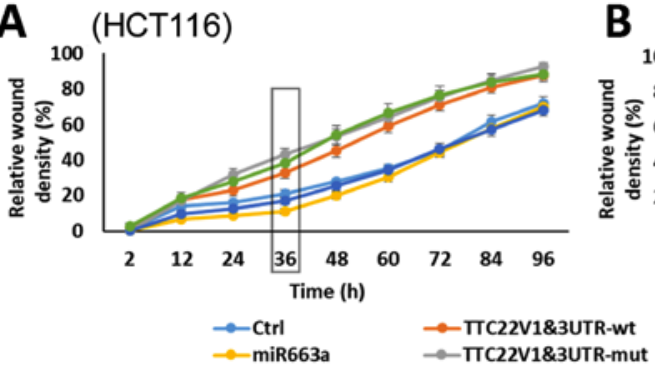

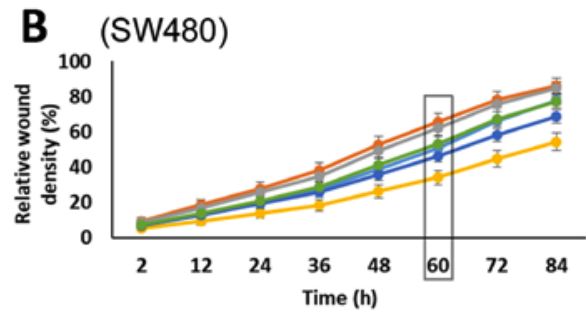

$\rightarrow-$ miR663a+TTC22V183UTR-wt $\rightarrow$ miR663a+TTC22V183UTR-mut

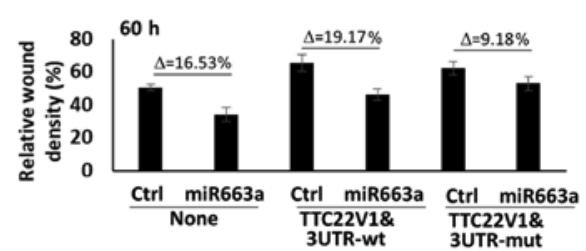

D
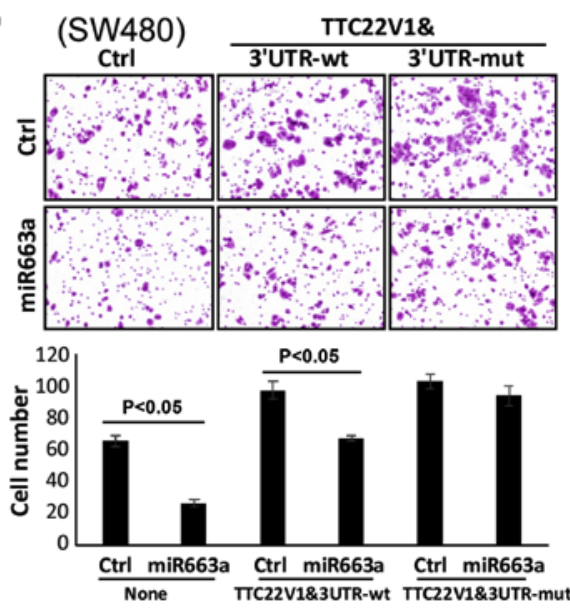

Figure 5. Effects of miR663a on the migration and invasion of colon cancer cells transiently transfected with wild-type TTC22V1 3'UTR and its mutant control. Migration curves for (A) HCT116 and (B) SW480 cells transiently co-transfected with the miR663a expression vector and TTC22V1\&3'UTR-wt or TTC22V1\&3'UTR-mut determined using long-term IncuCyte analyses. The cell density data for HCT116 cells at $36 \mathrm{~h}$ and for SW480 cells at $60 \mathrm{~h}$ are presented in the lower histograms. Each point represents the average value of 5-wells. Differences in the wound density between the miR663a and control groups of cells with and without TTC22V1\&3'UTR-wt/mut transfection are indicated. Typical Transwell analysis results for the invasion of (C) HCT116 and (D) SW480 cells co-transfected with the miR663a vector and TTC22V1\&3'UTR-wt or TTC22V1\&3'UTR-mut plasmids in triplicate. The migration times were $24 \mathrm{~h}$ for HCT116 cells and $36 \mathrm{~h}$ for SW480 cells. miR663a, microRNA-663a; TTC22V1, tetratricopeptide repeat domain 22 variant 1; TTC22V1\&3'UTR-wt, TTC22V1 cDNA sequence containing a wild-type 3'UTR; TTC22V1\&3'UTR-mut, TTC22V1 cDNA sequence containing a 3'UTR mutant; Ctrl, pcDNA3.1b scramble RNA control vector.

IHC staining demonstrated that the TTC22V1 protein was located principally in the nuclei of epithelial cells at the crypt base (or stem cell niche) in the SM mucosa samples (Fig. 6D), but no TTC22V1 staining signals were observed in the CC samples (Fig. 6E). Only weak (if any) cytoplasmic TTC22V1 staining was detected in the $\mathrm{CC}$ cells. According to the immunofluorescence confocal microscopy assays, TTC22V1 protein staining signals could be observed in the nuclei and cytoplasm in HCT116 and Caco2 cells, but only in the nucleus in SW480 (Fig. S3). Nuclear speckles enriched with endogenous TTC22 protein were also frequently observed in these cells.

Notably, more TTC22V1 mRNA was detected in metastatic CC compared with in non-metastatic CC (Mann-Whitney $\mathrm{U}$ test; $\mathrm{P}=0.022$ for lymph metastasis and 0.002 for distant metastasis; Table I). According to the Kaplan-Meier survival analysis, the overall survival time was significantly shorter for patients with CC with high TTC22Vl expression compared with for those with low TTC22VI expression (log-rank test;
$\mathrm{P}=0.023$; Fig. 6F). High TTC22V1 expression remained a significant poor survival factor following adjustment for age, sex, location, depth of invasion, differentiation, vascular embolism, lymph metastasis and distant metastasis (adjusted-hazard ratio, 1.765; 95\% confidence interval, 1.013-3.076; $\mathrm{P}=0.045$ ). Collectively, these results suggest that TTC22V1 may be a metastasis-associated gene and crucial miR663a target.

\section{Discussion}

Primate-specific miR663a is a rare gene located in the sequence flanking the centromere of chromosome 20. It is well-known that miR663a is an inflammation-associated miRNA (1-6) and that local chronic inflammation contributes to colorectal cancer (25-29). However, it is unknown whether alterations in miR663a expression contribute to CC development and progression. Conflicting results in previous studies suggest that the effects of miR663a on cancer development and progression may be tissue/organ-dependent (7-12). In 

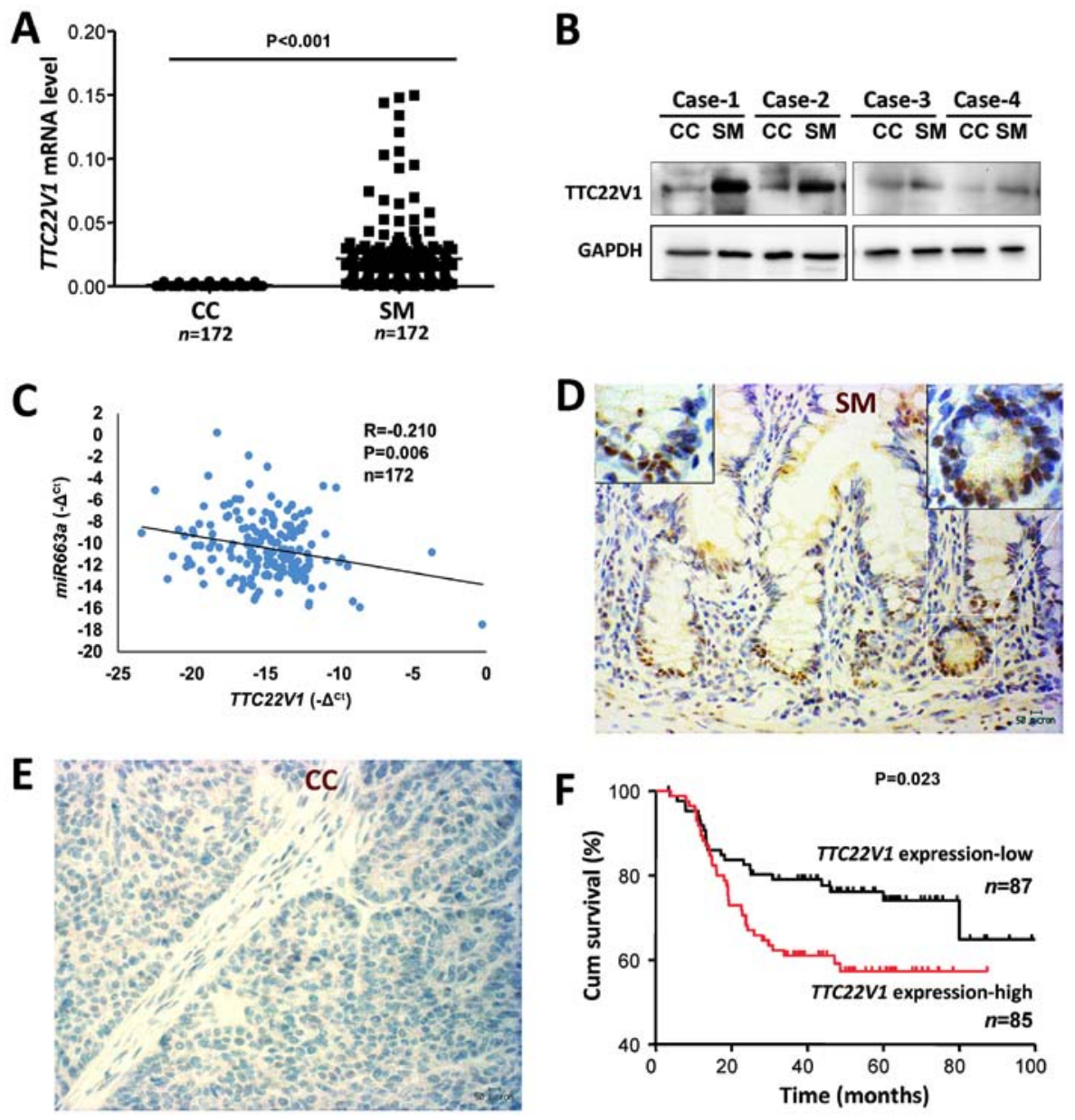

Figure 6. Downregulation of TTC22Vl expression associated with CC metastasis. (A) Comparison of TTC22V1 expression levels in CC and paired SM samples using the reverse transcription-quantitative polymerase chain reaction. (B) Comparison of TTC22VI expression levels in CC and paired SM samples using western blot analysis. (C) Association between TTC22V1 and miR663a RNA levels in 172 CC tissues using ALU and U6 as reference genes. (D) TTC22V1 staining; 2 crypt bases with nucleic TTC22-positive epithelial cells in representative SM tissues are presented in the insets. (E) No TTC22 staining signals were observed in paired CC tissues. (F) Kaplan-Meier survival curves for patients with CC classified into high and low TTC22V1 expression groups. The cut-off value to define high TTC22V1 expression samples was $>0.0008$. TTC22, tetratricopeptide repeat domain 22; TTC22V1, TTC22 variant 1; CC, colon cancer; SM, surgical margin; miR663a, microRNA-663a; Cum, cumulative.

the present study, it was identified that miR663a expression was downregulated during $\mathrm{CC}$ development and that low miR663a expression was associated with a high risk of $\mathrm{CC}$ metastasis. The in vitro and in vivo experimental results suggest that miR663a is a suppressor of CC progression. Most importantly, it was identified, for the first time, to the best of our knowledge, that TTC22V1 mRNA is a crucial miR663a target in CC cells. TTC22V1 significantly increased CC cell migration and invasion, and the risk of CC metastasis, and decreased patient survival times. These results indicate that the miR663a-TTC22V1 axis may serve an important role in CC metastasis.

Currently, there is no information on the biological function of the TTC22 gene. The bioinformatics analysis of the Cancer Cell Line Encyclopedia data revealed that TTC22V1 shared the most co-expressed genes with $C D H 1$ in human cell lines ( $n=1037$; data not shown) (30). According to publicly available transcriptome databases for various human tissues and the Protein Atlas (20,31), TTC22 is markedly expressed in the gastrointestinal epithelium, female organs and skin, which are frequently exposed to environmental factors. Furthermore, TTC22VI expression is extensively downregu- lated in a number of types of cancer. The IHC analysis of the present study demonstrated further that the TTC22 protein is located principally within the nuclei of epithelial cells at normal colon crypt base stem cell niches and that nucleic TTC22 is lacking in the majority of CC tissues. RT-qPCR and western blot results confirmed considerably lower TTC22V1 expression in CC tissues compared with in SM tissues. These results imply that TTC22 is involved in maintaining regular epithelium regeneration in the colon mucosa and the intense self-renewal kinetics of intestinal stem cells. Notably, TTC22V1 mRNA levels were significantly higher in metastatic CC compared with in non-metastatic CC, and forced TTC22V1 overexpression increased CC cell migration and invasion. The overall survival times of patients with $\mathrm{CC}$ with high TTC22V1 mRNA levels was much shorter compared with that of patients with low TTC22VI expression. Similar survival differences were also identified in several public databases of patients with CC (bioinformatica.mty.itesm. mx:8080/Biomatec/SurvivaX.jsp) (32-34) (Fig. S4). These results suggest that $T T C 22 \mathrm{VI}$ is required for CC cell migration and subsequent metastasis. It is worthwhile to investigate whether nucleic TTC22V1 is essential for the shift in regener- 
ating cells from the base crypt to the mucosa surface and the migration of CC cells.

Generally, the majority of mature miRNAs bind to the target mRNA 3'UTR and 5'UTR in the cytoplasm, leading to target mRNA degradation or translation inhibition. The functions of miRNAs are largely dependent on the expression patterns of target genes in the cells. Except for TTC22V1, however, expression changes in other miR663a target genes were not detected in HCT116 cells with miR663a overexpression or downregulation in the present study. It was also determined that miR663a overexpression could significantly inhibit migration and invasion in CC cells overexpressing TTC22V1 containing the 3'UTR-wt sequence, but only slightly inhibit migration and invasion in cells overexpressing the 3'UTR-mut counterpart. These results provide direct evidence to support TTC22V1 being a crucial factor for inhibiting the migration and invasion of CC cells by miR663a.

In conclusion, the results of the present study revealed that TTC22V1 mRNA is a crucial miR663a target. Whereas miR663a suppresses CC metastasis, TTC $22 \mathrm{Vl}$ promotes CC metastasis. The miR663a-TTC22Vl axis may serve an important role in $\mathrm{CC}$ metastasis and thus be a target for preventing $\mathrm{CC}$ progression.

\section{Acknowledgements}

The authors thank Dr Xin Bi from the University of Pennsylvania (Philadelphia, PA, USA) for her writing assistance.

\section{Funding}

The present study was supported by the National Natural Science Foundation of China (grant no. 81372592).

\section{Availability of data and materials}

The datasets used during the present study are available from the corresponding author upon reasonable request.

\section{Authors' contributions}

WT, YD, YM and BZ analyzed gene interactions, and interpreted the patient data regarding gene expression and colon cancer prognosis. LG and JZ participated in the collection of cancer tissues and determination of gene expression levels. WT, YD and DD designed these experiments and were major contributors to writing the manuscript. DD conceived the idea for the study and supervised the entire project. All authors read and approved the final manuscript.

\section{Ethics approval and consent to participate}

The Peking University Cancer Hospital and Institutional Review Boards approved this study. All patients provided written informed consent to participate in the study. Ethical approval for the animal experiments was obtained.

\section{Patient consent for publication}

Not applicable.

\section{Competing interests}

The authors declare that they have no competing interests.

\section{References}

1. Dreher A, Rossing M, Kaczkowski B, Nielsen FC and Norrild B: Differential expression of cellular microRNAs in HPV-11 transfected cells. An analysis by three different array platforms and qRT-PCR. Biochem Biophys Res Commun 403: 357-362, 2010.

2. Liang P, Lv C, Jiang B, Long X, Zhang P, Zhang M, Xie T and Huang X: MicroRNA profiling in denatured dermis of deep burn patients. Burns 38: 534-540, 2012.

3. Li P,Zhu N, YiB, Wang N, Chen M, You X, Zhao X, Solomides CC, Qin Y and Sun J: MicroRNA-663 regulates human vascular smooth muscle cell phenotypic switch and vascular neointimal formation. Circ Res 113: 1117-1127, 2013.

4. Miao CG, Shi WJ, Xiong YY, Yu H, Zhang XL, Qin MS, Du CL, Song TW, Zhang B and Li J: MicroRNA-663 activates the canonical Wnt signaling through the adenomatous polyposis coli suppression. Immunol Lett 166: 45-54, 2015.

5. Hu W, Xu S, Yao B, Hong M, Wu X, Pei H, Chang L, Ding N, Gao X, Ye C, et al: MiR-663 inhibits radiation-induced bystander effects by targeting TGFB1 in a feedback mode. RNA Biol 11: 1189-1198, 2014.

6. Latruffe N, Lançon A, Frazzi R, Aires V, Delmas D, Michaille JJ, Djouadi F, Bastin J and Cherkaoui-Malki M: Exploring new ways of regulation by resveratrol involving miRNAs, with emphasis on inflammation. Ann NY Acad Sci 1348: 97-106, 2015.

7. Jiao L, Deng Z, Xu C, Yu Y, Li Y, Yang C, Chen J,Liu Z, Huang G, Li LC, et al: miR-663 induces castration-resistant prostate cancer transformation and predicts clinical recurrence. J Cell Physiol 229: 834-844, 2014.

8. Yi C, Wang Q, Wang L, Huang Y, Li L, Liu L, Zhou X, Xie G, Kang $\mathrm{T}$, Wang $\mathrm{H}$, et al: MiR-663, a microRNA targeting p21 WAFI/CIPI , promotes the proliferation and tumorigenesis of nasopharyngeal carcinoma. Oncogene 31: 4421-4433, 2012.

9. Liang S, Zhang N, Deng Y, Chen L, Zhang Y, Zheng Z, Luo W, Lv Z, Li S and Xu T: miR-663 promotes NPC cell proliferation by directly targeting CDKN2A. Mol Med Rep 16: 4863-4870, 2017.

10. Shi Y, Chen C, Yu SZ, Liu Q, Rao J, Zhang HR, Xiao HL, Fu TW, Long H, He ZC, et al: miR-663 suppresses oncogenic function of CXCR4 in glioblastoma. Clin Cancer Res 21: 4004-4013, 2015.

11. Li Q, Cheng Q, Chen Z, Peng R, Chen R, Ma Z, Wan X, Liu J, Meng M, Peng Z, et al: MicroRNA-663 inhibits the proliferation, migration and invasion of glioblastoma cells via targeting TGF- $\beta 1$. Oncol Rep 35: 1125-1134, 2016.

12. Zang W, Wang Y, Wang T, Du Y, Chen X, Li M and Zhao G: miR-663 attenuates tumor growth and invasiveness by targeting eEF1A2 in pancreatic cancer. Mol Cancer 14: 37, 2015.

13. Tian W, Du Y,Ma Y, Gu L, Zhou J and Deng D: MALAT1-miR663a negative feedback loop in colon cancer cell functions through direct miRNA-lncRNA binding. Cell Death Dis 9: 857, 2018.

14. Huang W, Li J, Guo X, Zhao Y and Yuan X: miR-663a inhibits hepatocellular carcinoma cell proliferation and invasion by targeting HMGA2. Biomed Pharmacother 81: 431-438, 2016.

15. Afonyushkin T, Oskolkova OV and Bochkov VN: Permissive role of miR-663 in induction of VEGF and activation of the ATF4 branch of unfolded protein response in endothelial cells by oxidized phospholipids. Atherosclerosis 225: 50-55, 2012.

16. Tili E, Michaille JJ, Adair B, Alder H, Limagne E, Taccioli C, Ferracin M, Delmas D, Latruffe N and Croce CM: Resveratrol decreases the levels of miR-155 by upregulating miR-663, a microRNA targeting JunB and JunD. Carcinogenesis 31: 1561-1566, 2010

17. Acevedo N, Reinius LE, Vitezic M, Fortino V, Söderhäll C, Honkanen H, Veijola R, Simell O, Toppari J, Ilonen J, et al: Age-associated DNA methylation changes in immune genes, histone modifiers and chromatin remodeling factors within 5 years after birth in human blood leukocytes. Clin Epigenetics 7: 34, 2015.

18. Allan RK and Ratajczak T: Versatile TPR domains accommodate different modes of target protein recognition and function. Cell Stress Chaperones 16: 353-367, 2011.

19. Blatch GL and Lässle M: The tetratricopeptide repeat: A structural motif mediating protein-protein interactions. BioEssays 21: 932-939, 1999. 
20. Uhlén M, Fagerberg L, Hallström BM, Lindskog C, Oksvold P, Mardinoglu A, Sivertsson A, Kampf C, Sjöstedt E, Asplund A, et al: Proteomics. Tissue-based map of the human proteome. Science 347: 1260419, 2015.

21. Sobin LH and Wittekind Ch (eds): International Union Against Cancer (UICC): TNM classification of malignant tumors. 6th edition. Wiley, New York, 2002

22. Marullo M, Zuccato C, Mariotti C, Lahiri N, Tabrizi SJ, Di Donato S and Cattaneo E: Expressed Alu repeats as a novel, reliable tool for normalization of real-time quantitative RT-PCR data. Genome Biol 11: R9, 2010.

23. Livak KJ and Schmittgen TD: Analysis of relative gene expression data using real-time quantitative PCR and the $2^{-\Delta \Delta C_{\mathrm{T}}}$ method. Methods 25: 402-408, 2001.

24. Tian W, Qu L, Meng L, Liu C, Wu J and Shou C: Phosphatase of regenerating liver-3 directly interacts with integrin $\beta 1$ and regulates its phosphorylation at tyrosine 783 . BMC Biochem 13 22, 2012.

25. Ullman TA and Itzkowitz SH: Intestinal inflammation and cancer. Gastroenterology 140: 1807-1816, 2011.

26. Liu L, Nishihara R, Qian ZR, Tabung FK, Nevo D, Zhang X, Song M, Cao Y, Mima K, Masugi Y, et al: Association between inflammatory diet pattern and risk of colorectal carcinoma subtypes classified by immune responses to tumor. Gastroenterology 153: 1517-1530.e14, 2017.

27. Shivappa N, Godos J, Hébert JR, Wirth MD, Piuri G, Speciani AF and Grosso G: Dietary inflammatory index and colorectal cancer risk - a meta-analysis. Nutrients 9: 9, 2017.

28. Pastille E, Frede A, McSorley HJ, Gräb J, Adamczyk A, Kollenda S, Hansen W, Epple M, Buer J, Maizels RM, et al: Intestinal helminth infection drives carcinogenesis in colitis-associated colon cancer. PLoS Pathog 13: e1006649, 2017.
29. Biswas S, Davis H, Irshad S, Sandberg T, Worthley D and Leedham S: Microenvironmental control of stem cell fate in intestinal homeostasis and disease. J Pathol 237: 135-145, 2015.

30. Consortium CCLE; Cancer Cell Line Encyclopedia Consortium; Genomics of Drug Sensitivity in Cancer Consortium: Pharmacogenomic agreement between two cancer cell line data sets. Nature 528: 84-87, 2015

31. Uhlen M, Zhang C, Lee S, Sjöstedt E, Fagerberg L, Bidkhori G, Benfeitas R, Arif M, Liu Z, Edfors F, et al: A pathology atlas of the human cancer transcriptome. Science 357: 357, 2017.

32. Aguirre-Gamboa R, Gomez-Rueda H, Martínez-Ledesma E, Martínez-Torteya A, Chacolla-Huaringa R, RodriguezBarrientos A, Tamez-Peña JG and Treviño V: SurvExpress: An online biomarker validation tool and database for cancer gene expression data using survival analysis. PLoS One 8: e74250, 2013.

33. Loboda A, Nebozhyn MV, Watters JW, Buser CA, Shaw PM, Huang PS, Van't Veer L, Tollenaar RA, Jackson DB, Agrawal D, et al: EMT is the dominant program in human colon cancer. BMC Med Genomics 4: 9, 2011.

34. Sveen A, Agesen TH, Nesbakken A, Rognum TO, Lothe RA and Skotheim RI: Transcriptome instability in colorectal cancer identified by exon microarray analyses: Associations with splicing factor expression levels and patient survival. Genome Med 3: 32, 2011.

This work is licensed under a Creative Commons Attribution-NonCommercial-NoDerivatives 4.0 International (CC BY-NC-ND 4.0) License. 